\title{
BMJ Open Efficacy and safety of the glucagon-like peptide-1 receptor agonist liraglutide added to insulin therapy in poorly regulated patients with type 1 diabetes - a protocol for a randomised, double-blind, placebo-controlled study: The Lira-1 study
}

Thomas Fremming Dejgaard, ${ }^{1,2}$ Filip Krag Knop, ${ }^{3,4}$ Lise Tarnow, ${ }^{5}$ Christian Seerup Frandsen, ${ }^{2}$ Tanja Stenbæk Hansen, ${ }^{1}$ Thomas Almdal, ${ }^{3}$ Jens Juul Holst, ${ }^{4}$ Sten Madsbad, ${ }^{2}$ Henrik Ullits Andersen ${ }^{1}$

To cite: Dejgaard TF, Knop FK, Tarnow L, et al. Efficacy and safety of the glucagon-like peptide-1 receptor agonist liraglutide added to insulin therapy in poorly regulated patients with type 1 diabetes-a protocol for a randomised, doubleblind, placebo-controlled study: The Lira-1 study. BMJ Open 2015;5:e007791. doi:10.1136/bmjopen-2015007791

- Prepublication history for this paper is available online. To view these files please visit the journal online (http://dx.doi.org/10.1136/ bmjopen-2015-007791).

Received 28 January 2015 Revised 12 March 2015 Accepted 17 March 2015

CrossMark

For numbered affiliations see end of article.

Correspondence to

Thomas Fremming Dejgaard; tfde@steno.dk

\section{ABSTRACT}

Introduction: Intensive insulin therapy is recommended for the treatment of type 1 diabetes (T1D). Hypoglycaemia and weight gain are the common side effects of insulin treatment and may reduce compliance. In patients with insulin-treated type 2 diabetes, the addition of glucagon-like peptide-1 receptor agonist (GLP-1RA) therapy has proven effective in reducing weight gain and insulin dose. The present publication describes a protocol for a study evaluating the efficacy and safety of adding a GLP-1RA to insulin treatment in overweight patients with T1D in a randomised, double-blinded, controlled design.

Methods and analysis: In total, 100 patients with type 1 diabetes, poor glycaemic control (glycated haemoglobin (HbA1c) $>8 \%$ ) and overweight (body mass index $>25 \mathrm{~kg} / \mathrm{m}^{2}$ ) will be randomised to either liraglutide $1.8 \mathrm{mg}$ once daily or placebo as an add-on to intensive insulin therapy in this investigator initiated, doubleblinded, placebo-controlled parallel study. The primary end point is glycaemic control as measured by changes in HbA1c. Secondary end points include changes in the insulin dose, hypoglyacemic events, body weight, lean body mass, fat mass, food preferences and adverse events. Glycaemic excursions, postprandial glucagon levels and gastric emptying rate during a standardised liquid meal test will also be studied.

Ethics and dissemination: The study is approved by the Danish Medicines Authority, the Regional ScientificEthical Committee of the Capital Region of Denmark and the Data Protection Agency. The study will be carried out under the surveillance and guidance of the good clinical practice (GCP) unit at Copenhagen University Hospital Bispebjerg in accordance with the ICH-GCP guidelines and the Helsinki Declaration.

Trial registration number: NCT01612468.

\section{Strengths and limitations of this study}

- A randomised, double-blinded, placebo-controlled design.

- After 24 weeks of intervention, it is possible to draw conclusions on glycated haemoglobin, glycaemic variability and weight in a glycaemic steady state.

- The study may not be sufficiently powered to draw on all secondary end points.

- These overweight and poorly controlled participants represent a subgroup of patients with type 1 diabetes.

\section{INTRODUCTION}

Patients with type 1 diabetes (T1D) are most often treated either by insulin injection regimens, including long-acting and short-acting insulin (basal/bolus therapy) or by insulin pump therapy. ${ }^{1}$ Several studies have demonstrated that an intensified insulin regimen in T1D can delay the onset and slow the progression of microvascular complications and may potentially reduce the long-term risk of macrovascular disease. ${ }^{2}{ }^{3}$ Based on this evidence, intensive insulin treatment aiming at near-normalisation of blood glucose levels with a glycated haemoglobin (HbA1c) of $<7.0 \%$ is recommended. ${ }^{4}$

Intensive insulin therapy with nearnormalised glycaemic control is, however, associated with side effects such as weight gain and risk of hypoglycaemia. ${ }^{2}$ Severe hypoglycaemic episodes are associated with 
serious physical and psychological morbidity and, occasionally, death. ${ }^{5}$ Episodes of hypoglycaemia can also result in hypoglycaemia unawareness and have been shown to cause significant anxiety and fear of future hypoglycaemic episodes, which may result in reduced compliance with treatment. Therefore, hypoglycaemia constitutes a major limiting factor in obtaining strict glycaemic control. ${ }^{6}$ Weight gain has also been reported during intensive insulin treatment. This may potentially lead to increased risk of overweight-related comorbidities $^{7}$ and result in non-compliance with intensive insulin treatment resulting in poor glycaemic control. Presently, approximately $50 \%$ of patients with T1D in high-income countries are overweight. ${ }^{8}$ Basal insulin alone cannot control postprandial glucose (PPG) excursions. As a result, patients with T1D are dependent on boluses of rapid-acting insulin at each meal. The aim of this is to correct any premeal hyperglycaemia and match the estimated carbohydrate content of the meal, also taking into account physical activity. For many patients, this can be a complicated task and is often associated with either hypoglycaemic or hyperglycaemic excursions, which may be accompanied by resulting emotional effects, resulting in poor glycaemic control and overall quality of life. Given these limiting factors, there is a need for a more convenient treatment regimen, which can improve glycaemic control, yet limit the side effects of, and problems associated with, current insulin regimens, namely hypoglycaemia, weight gain and exaggerated PPG excursions. $^{9}$ Risk of hypoglycaemia and weight gain is also evident in insulin-treated patients with type 2 diabetes (T2D). ${ }^{10}{ }^{11}$ However, the combination of insulin with a glucagon-like peptide-1 receptor agonist (GLP-1RA) has proven effective in reducing the weight gain and insulin dose in insulin-treated patients with T2D without a deterioration in glycaemic control or increased risk of hypoglycaemia. ${ }^{12} 13$

In mechanistic studies, GLP-1RA improved glycaemic control by delayed gastric emptying, ${ }^{14}{ }^{15}$ supressed postprandial glucagon, ${ }^{14}{ }^{15}$ enhanced insulin secretion in $\mathrm{T}_{2} \mathrm{D}^{16}$ and C-peptide positive patients with $\mathrm{T}^{1} \mathrm{D}^{14}$ and reduced postprandial glycaemic excursions. ${ }^{16} 17$

Few clinical trials have assessed the safety and efficacy of GLP-1RA treatment in patients with T1D. However, a few short-term studies have demonstrated weight loss ranging between 2.8 and $4.5 \mathrm{~kg}$, fewer PPG excursions and reduced insulin requirements $(0.07-0.19 \mathrm{U} / \mathrm{kg} /$ day $)$, concomitant with improved or unaltered glycaemic control. ${ }^{14} \quad 15 \quad 18-23$ The dose-response relationship has been evaluated, and a preliminary interim analysis showed that the addition of 1.2 and $1.8 \mathrm{mg}$ liraglutide significantly reduced $\mathrm{HbAlc}$, mean blood glucose and body weight in overweight people with T1D compared to placebo. ${ }^{24}$ Based on this background, it would seem relevant to evaluate the efficacy and safety of long-term (24 weeks) GLP-1RA treatment with $1.8 \mathrm{mg}$ liraglutide in patients with T1D in a randomised, controlled and double-blinded study design. The protocol for this is described in this paper.

\section{HYPOTHESIS}

Treatment with the GLP-1RA liraglutide as an add-on to the existing basal/bolus intensive insulin therapy in poorly regulated $(\mathrm{HbAlc}>8.0 \%)$ and overweight (body mass index $>25 \mathrm{~kg} / \mathrm{m}^{2}$ ) patients with T1D results in reductions in $\mathrm{HbA1c}$, body weight, insulin dose, glycaemic variability, hypoglycaemic events and body fat mass.

\section{OBJECTIVES AND END POINTS}

The objective of the present protocol is to evaluate the effect of liraglutide as an adjunctive therapy to intensive insulin treatment in overweight and poorly regulated patients with T1D.

The primary end point is metabolic control as measured by changes in HbAlc from baseline. Secondary end points include change in insulin dose, hypoglycaemic events, glycaemic variability measured by continuous glucose monitoring (CGM), body weight, lean body mass and fat mass as determined by a dual-energy X-ray absorptiometry (DXA) scan, food preferences, appetite score, quality of life, treatment satisfaction and adverse events. In addition, postprandial glucagon and GLP-1 levels, gastric emptying and glycaemic excursions will be estimated during a standardised liquid mixed meal test (MMT). Primary and secondary end points are shown in box 1.

\section{METHODS AND ANALYSES \\ Research design}

The study is an investigator-initiated, randomised, double-blinded, placebo-controlled intervention trial. In total, 100 patients with T1D treated with a basal/bolus insulin regimen will be randomised in a 1:1 ratio to liraglutide $1.8 \mathrm{mg}$ once daily, or placebo as an add-on to insulin therapy. Treatment allocation will be generated and data maintained in a web-based case report file (eCRF). If necessary, unblinding can be made individually so that treatment blinding of other patients in the study remains.

\section{Study population}

Patients will be recruited from outpatient clinics in the Capital Region of Denmark by the investigator. All patients who meet the inclusion and exclusion criteria at screening will be enrolled for randomisation followed by a 24-week treatment period at the Steno Diabetes Center, Gentofte, Denmark. The inclusion and exclusion criteria are listed in box 2 .

\section{Trial visits and examinations}

Patients will receive written and oral information about the study. Sufficient time will be given before written informed consent is obtained by the investigator. At screening (visit 0), demography, medical history and concomitant medication will be recorded. A physical examination including heart rate, blood pressure (BP) and ECG will be performed together with blood 


\section{Box 1 Outcome measures}

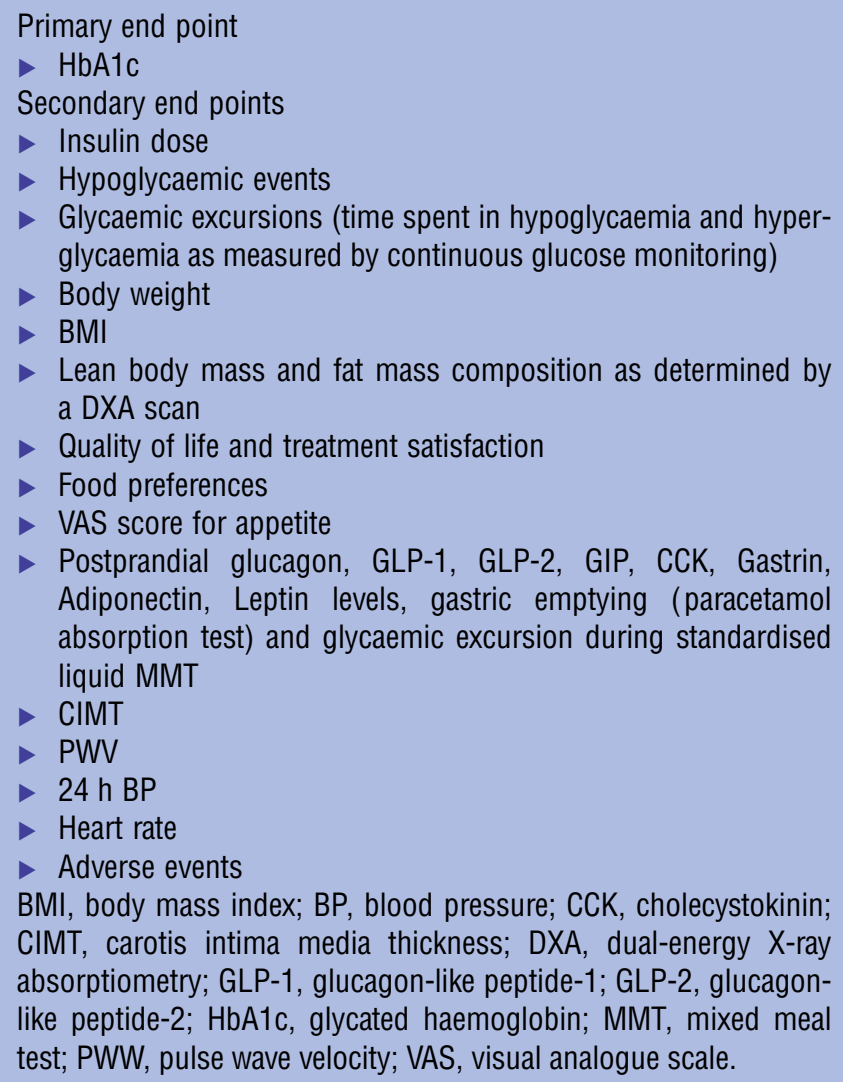

samples and urine tests. Six days of CGM will be performed prior to randomisation (visit 1), visit 3 (week 14) and at the end of treatment (week 26). An overview of blood and urine samples is shown in table 1 .

During the trial, the insulin dose will be adjusted according to the self-measured blood glucose, CGM and HbAlc. Treatment targets for BG will be preprandial values of $4-7 \mathrm{mmol} / \mathrm{L}$ and postprandial values of $<10 \mathrm{mmol} / \mathrm{L}$ according to the Danish guidelines for the treatment of T1D. ${ }^{25}$ At randomisation, the insulin dose will be reduced by $33 \%$ for the bolus and $25 \%$ for basal insulin before study drug initiation to reduce the risk of hypoglycaemia. Furthermore, patients will undergo physical examination, have blood and urine samples taken, have a DXA scan, and complete questionnaires concerning quality of life (WHO-5) and problem areas in diabetes (PAID), as well as the Diabetes Treatment and Satisfaction Questionnaire (DTSQ). Carotid intima media thickness and pulse wave velocity scans will be determined together with a $24 \mathrm{~h} \mathrm{BP}$ evaluation before and at the end of treatment with the study drug.

A telephone contact to adjust insulin dosing and ensure compliance and evaluation of adverse effects will be performed between visit 1 and visit 2 . The patients will be advised to contact the study team if they encounter any problems with the dosing of insulin and/or if glucose control occurs.
Box 2 Overview of inclusion and exclusion criteria

Inclusion criteria

- T1D (according to the WHO criteria) $\geq 1$ year

- Age $\geq 18$ years

- $\mathrm{BMI}>25.0 \mathrm{~kg} / \mathrm{m}^{2}$

- $\mathrm{HbA} 1 \mathrm{C}>8.0 \%$ at visit 0

- Able to understand the written patient information and to give informed consent

Exclusion criteria

- Insulin pump treatment

- Hypoglycaemia unawareness

- Diabetic gastroparesis

- Impaired kidney function (eGFR $<60 \mathrm{~mL} / \mathrm{min} / 1.73 \mathrm{~m}^{2}$ ), dialysis or kidney transplantation

- Liver disease with elevated plasma ALT >three times the upper normal limit

- Acute or chronic pancreatitis

- Inflammatory bowel disease

- Cancer unless in complete remission for $>5$ years

- History of thyroid adenoma or carcinoma

- Other concomitant disease or treatment that according to the investigator's assessment makes the patient unsuitable for study participation

- Alcohol and/or drug abuse

- Fertile women not using chemical or mechanical contraceptives

- Pregnant or nursing women

- Known or suspected hypersensitivity to trial product or related products

- Receipt of an investigational drug within 30 days prior to visit 0

- Simultaneous participation in any other clinical intervention trial

ALT, alanine aminotransferase; eGFR, estimated glomerular filtration rate; T1D, type 1 diabetes.

At visit 1 (randomisation), visit 2 (clinical control) and visit 5 (end of treatment), the first 40 enrolled patients will undergo a $4 \mathrm{~h}$ MMT measuring postprandial glucagon levels, endogenous GLP-1 and gastric emptying evaluated by the paracetamol absorption test. Visual analogue scale (VAS) tests for appetite and satiety measures and a questionnaire concerning food preferences will also be performed during each MMT.

At all visits, adverse events, current medication, body weight, basal and bolus insulin doses and hypoglycaemic events will be recorded. Additionally, BP and heart rate will be measured. Urinary human chorionic gonadotropin will be measured in women of childbearing potential.

A flow chart and an outline of the trial visits and examinations are shown in figure 1 and table 2.

\section{INTERVENTION}

Name: Victoza (liraglutide) or matching placebo.

Pharmaceutical form: Liraglutide $6.0 \mathrm{mg} / \mathrm{mL}, 3 \mathrm{~mL}$ prefilled pen for subcutaneous injection. Placebo, $3 \mathrm{~mL}$ prefilled pen for subcutaneous injection.

Pharmaceutical dosage: Liraglutide or placebo will be initiated at a dose of $0.6 \mathrm{mg} /$ day and gradually escalated up to $1.2 \mathrm{mg}$ /day after 1 week and after another week to $1.8 \mathrm{mg} /$ day. Intervals between dose increments can be 


\begin{tabular}{|c|c|}
\hline Sampling period & Analysis \\
\hline Screening and control visits & $\begin{array}{l}\text { Glucose, HbA1c } \\
\text { C-peptide, GAD antibodies } \\
\text { Haemoglobin, } \\
\text { thrombocytes, } \\
\text { leucocytes } \\
\text { Albumin } \\
\text { Creatinine, potassium, } \\
\text { sodium } \\
\text { TSH } \\
\text { Cholesterol, triglycerides } \\
\text { ALT, lipase, amylase } \\
\text { Urine albumin-creatinine } \\
\text { ratio, hCG }\end{array}$ \\
\hline MMT & $\begin{array}{l}\text { Paracetamol } \\
\text { Glucose, C-peptide } \\
\text { Glucagon } \\
\text { GLP-1, GLP-2, GIP } \\
\text { Adiponectin, leptin } \\
\text { CCK, Gastrin } \\
\text { NT-proBNP } \\
\text { hsCRP }\end{array}$ \\
\hline \multicolumn{2}{|c|}{$\begin{array}{l}\text { ALT, alanine aminotransferase; CCK, cholecystokinin; GAD, } \\
\text { glutamic acid decarboxylase; GLP, glucagon-like peptide; HbA1c, } \\
\text { glycated haemoglobin; hCG, human chorionic gonadotropin; } \\
\text { hsCRP, high-sensitive C reactive protein; MMT, mixed meal test; } \\
\text { NT-proBNP, N-terminal prohormone brain natriuretic peptide; TSH, } \\
\text { thyroid-stimulating hormone. }\end{array}$} \\
\hline
\end{tabular}

extended based on the subject's tolerance to the trial product. Further, the trial drug dose can be reduced at any time during the trial if required. The injection can be given at any time of the day, but it is recommended that the time of injection is consistent from day to day.

Side effects: Common side effects (1-10\%): nausea, vomiting, diarrhoea, obstipation, hypoglycaemia and headache.

Shipping and packing: All trial products will be delivered, packed blinded and labelled by Novo Nordisk A/S.

\section{SAMPLE SIZE}

Sample size calculation is based on the ability to detect a difference in change from baseline in HbAlc (primary outcome) between study arms of $6 \mathrm{mmol} / \mathrm{mol}(0.6 \%)$ with $80 \%$ power, a $5 \%$-significance level and a presumed $9 \mathrm{mmol} / \mathrm{mol}(0.9 \%) \mathrm{SD}$. This will require 35 patients to be included in each study arm (two-sided test). To allow for a $30 \%$ dropout rate, 100 patients in total will be included in the study, 50 in each study arm. The sample size calculation is based on data obtained from a randomised controlled trial in patients with T1D treated with injection therapy versus insulin pump-therapy. ${ }^{26}$ Withdrawn participants will not be substituted.

\section{DATA ANALYSIS}

The per-protocol population will include all patients completing the study with a documented valid baseline and end-of-treatment (eventually last observation carried forward) assessment of the primary end point without any major protocol violations. The intention-to-treat population will include all randomised patients.

The primary efficacy outcome parameter is change in HbAlc from baseline to end of treatment between the intervention and the control group. The absolute values and adjusted mean changes from baseline for each treatment group, the difference in adjusted mean change between the liraglutide group and the placebo group, and the $95 \%$ CI will also be reported. The primary analysis will be based on the per-protocol population.

Comparisons between treatment groups will be performed by an unpaired two-sample t test, Mann-Whitney test or $\chi^{2}$ test as appropriate. Normally distributed variables will be presented as mean $\pm \mathrm{SD}$. Non-parametric statistics and appropriate log-transformation will be performed if assumption of normality is not met. A twotailed $p$ value of less than 0.05 will be considered statistically significant.
Figure 1 Flow chart of the study.

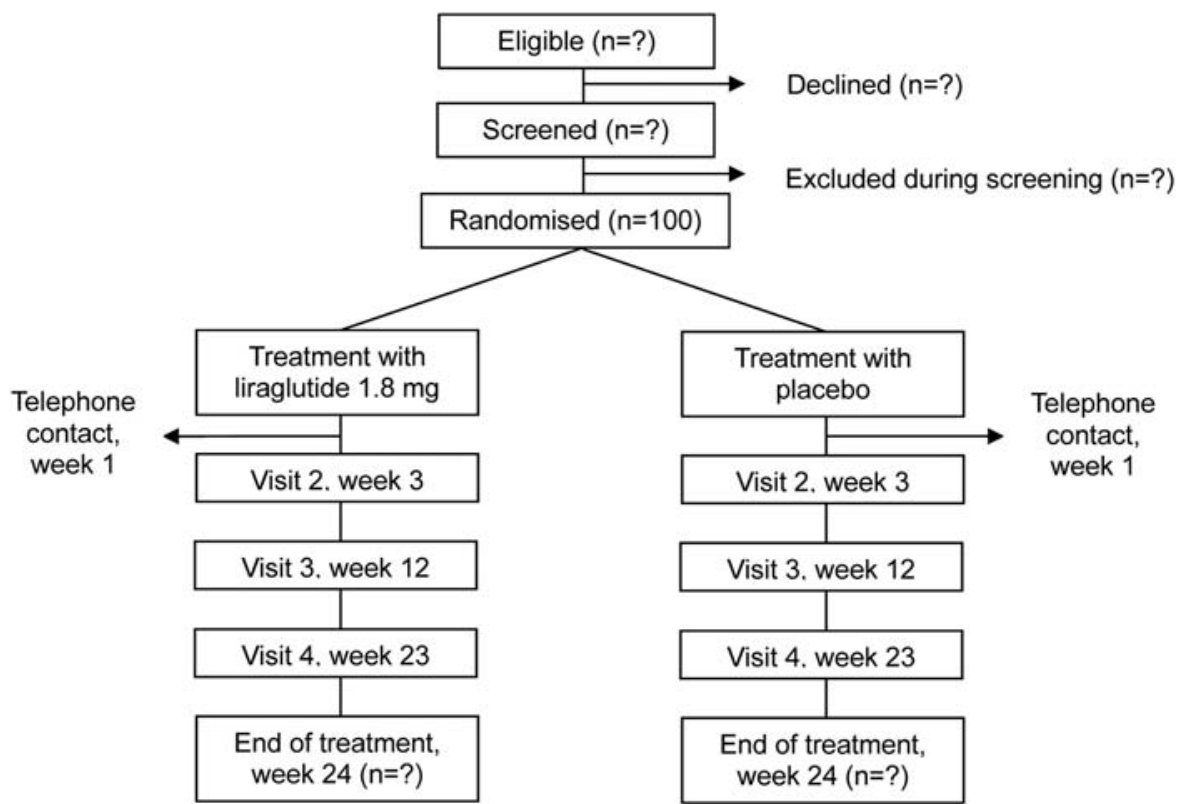




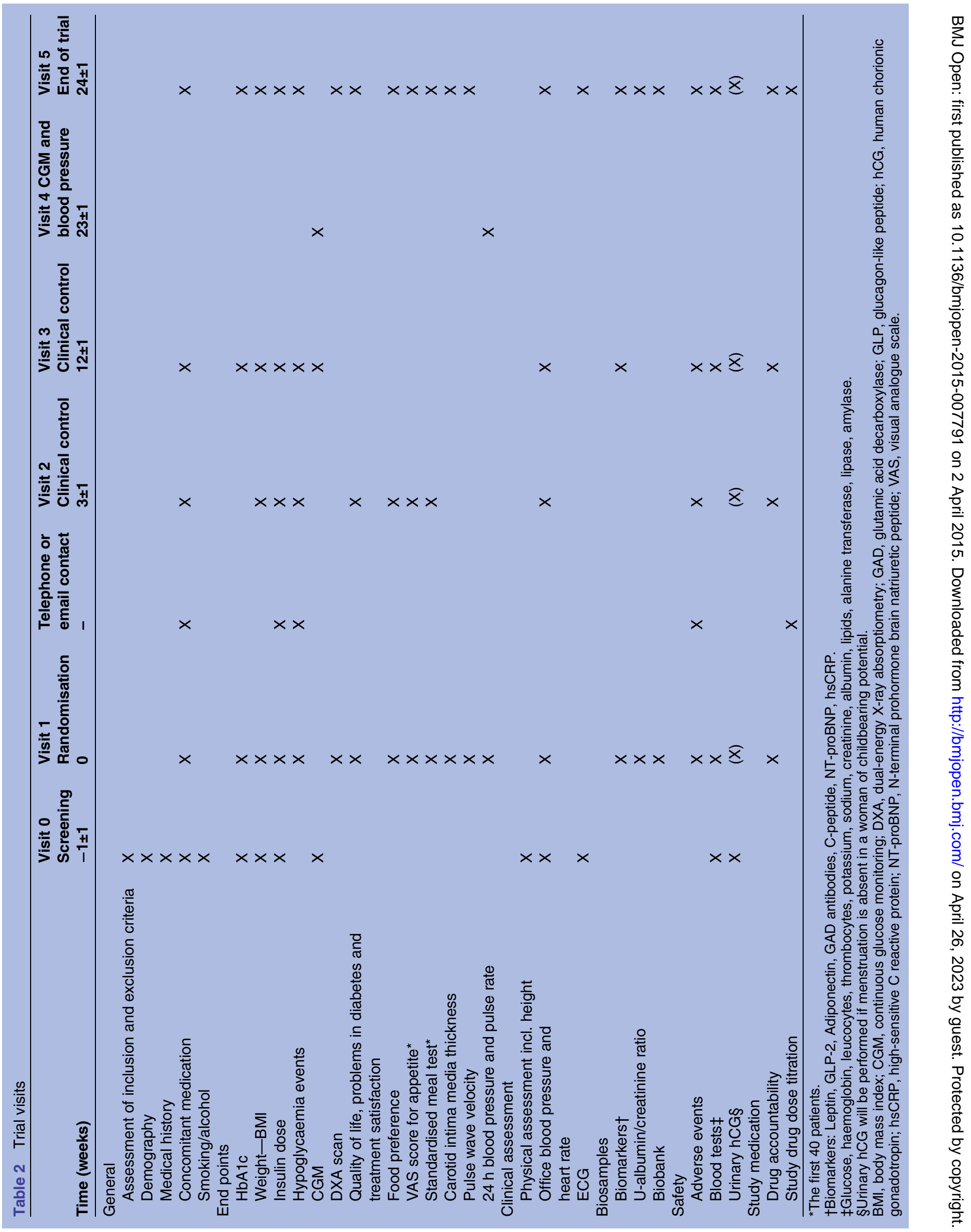


Additional analyses due to loss of follow-up include analysis of data from the intention-to-treat population to determine the validity of the conclusions of the perprotocol population and will include duration in study and the reason for discontinuation as covariables.

\section{ETHICS AND PUBLICATION}

It is expected that the study will contribute important and novel information about: treatment of patients with T1D with liraglutide as an add-on to insulin therapy; whether liraglutide treatment improves glycaemic control; and important safety information. A large number of patients worldwide could potentially benefit from this new treatment concept.

During the study, the patient will undergo frequent clinical check-ups by a physician. Therefore, the patient will be carefully evaluated with regard to safety and efficacy, and glucose regulation will be optimised continuously, which should reduce the risk of adverse events. Liraglutide (Victoza) is approved for treatment of T2D by the US Food and Drug Administration (FDA), State FDA (SFDA) China, Pharmaceuticals and Medical Devices Agency Japan (PMDA) and European Medicines Agency (EMA), and studies have shown limited adverse effects, that is, nausea, vomiting, obstipation and headache. Nausea and vomiting are usually transient and occur mostly during the first weeks after initiation of treatment. Adverse effects are generally temporary and can be minimised by gradual dose escalation, as planned in this study. In combination with insulin, the risk of hypoglycaemia can be minimised by reducing insulin doses at start of treatment and carefully instructing patients to monitor their blood glucose. For those receiving placebo, there will be a modest risk of hyperglycaemia, but patients can titrate insulin doses as needed, using frequent blood glucose measurements. Few cases of acute pancreatitis have been reported in relation to GLP-1 treatment, but overall the incidence did not differ from that in patients with T2D treated with other antidiabetic agents. ${ }^{27}$ Thyroid C-cell tumours have been seen in mice and rats treated with liraglutide, but have not been reported among participants treated with liraglutide in clinical studies.

At the two DXA scans, the patient will be exposed to a weak X-ray radiation, less than $1 \mathrm{mSv}$ in total. For comparison, the background radiation in Denmark is about $3 \mathrm{mSv}$ per year. The standardised meal test is preceded by $10 \mathrm{~h}$ of fasting, which may cause slight discomfort. A volume of $200 \mathrm{~mL}$ blood is taken during each meal test, and a total of $750 \mathrm{~mL}$ blood is taken during the 26-week period.

The results from the study will be presented at national and international scientific meetings, and the manuscripts will be written in accordance with the CONSORT 2010 statement and submitted to peer-reviewed journals. Novo Nordisk A/S has been allowed to comment on the protocol before initiation of the study and will have 4 weeks to comment on the manuscripts before publication.

\section{STUDY APPROVAL}

The Lira-1 study is approved by the Danish Medicines Agency (EudraCT number: 2012-001150-26), the Scientific-Ethical Committee of the Capital Region of Denmark (H-1-2012-031) and the Danish Data Protection Agency (SDC-2012-001). It is registered at ClinicalTrials. gov (NCT01612468) and will be carried out under the surveillance and guidance of the good clinical practice (GCP) unit at Copenhagen University Hospital in compliance with the ICH-GCP guidelines. The study was initiated in June 2012 and is expected to be reported in 2015. The study will be conducted in accordance with the Helsinki Declaration.

\section{DISCUSSION}

Insulin treatment of patients with T1D is often associated with weight gain and risk of hypoglycaemia. Many patients do not obtain the glycaemic goal despite basal/ bolus insulin therapy in combination with intensive selfmonitoring of blood glucose. ${ }^{8}$ Novel treatments addressing weight gain while improving glycaemic control are warranted; GLP-1 RAs appear promising in this regard.

The present protocol describes a randomised, doubleblinded, placebo-controlled trial evaluating the efficacy and safety of the long-acting GLP-1RA liraglutide as an add-on to insulin therapy in overweight and poorlycontrolled patients with T1D. The primary end point is change in HbAlc from baseline, but the trial will also address changes in glycaemic variability, weight, cardiovascular risk factors and PPG control and hormonal changes during an MMT. The trial will foremost give information on efficacy and safety of combination therapy as compared with insulin treatment alone.

As the duration of diabetes increases, endogenous insulin secretion is less important in patients with T1D than T2D, since no or very limited insulin secretion is present in T1D. ${ }^{28}$ However, other hormones such as glucagon, GLP-1, glucagon-like peptide-2 (GLP-2), gastric inhibitory peptide, cholecystokinin, gastrin and the peptide adiponectin and leptin could be influenced by GLP-1RA and might play an important role in appetite regulation in overweight patients with T1D. Of interest, the lowering of glucagon during a meal may be important for the regulation of PPG in patients with T1D. ${ }^{14}{ }^{29}$ Another mechanism of interest could be the effect of the reduction in the gastric emptying rate, resulting in delayed intestinal absorption of carbohydrates and therefore a reduction in PPG hyperglycaemia. $^{14} 30$ All of these outcomes will be addressed in the study as secondary end points.

The limitations of this trial are that the effects of combination therapy on weight and glycaemic control in normal weight patients with T1D are not addressed. Also, the effect of combination therapy on counterregulation during hypoglycaemia is not included in this protocol. A study investigating the effect on the gastric emptying rate and on glucagon response during hypoglycaemia is of high priority, since both a delaying in 
gastric emptying after food intake and an attenuated glucagon will compromise counter-regulation. ${ }^{19}$

In conclusion, the present study will elucidate many of the advantages and disadvantages associated with combination therapy with insulin and GLP-1RA in patients with T1D.

\section{Author affiliations}

${ }^{1}$ Steno Diabetes Center, Gentofte, Denmark

${ }^{2}$ Department of Endocrinology, Hvidovre Hospital, University of Copenhagen, Hvidovre, Denmark

${ }^{3}$ Center for Diabetes Research, Gentofte Hospital, University of Copenhagen, Hellerup, Denmark

${ }^{4}$ Faculty of Health and Medical Sciences, Department of Biomedical Sciences, The NNF Center for Basic Metabolic Research, University of Copenhagen, Copenhagen, Denmark

${ }^{5}$ Nordsjællands Hospital, University of Copenhagen, Hillerød, Denmark

Contributors TFD, FKK, TSH, LT, TA, JJH and HUA conceived and designed the study. HUA sponsors the trial and owns the data. TFD is the principal investigator. CSF is the subinvestigator. TFD, CSF, HUA, FKK and SM have drafted the manuscript. All authors have read and approved the final version of the manuscript.

Funding This work was initiated by the authors and is supported as an investigator-initiated study by an unrestricted grant from Novo Nordisk A/S.

Competing interests TFD, CSF and TSH have received research support from Novo Nordisk. FKK has received lecture fees from AstraZeneca, Boehringer Ingelheim Pharmaceuticals, Bristol-Myers Squibb, Eli Lilly and Company, Gilead Sciences, Merck Sharp \& Dohme, Novo Nordisk, Ono Pharmaceuticals, Sanofi and Zealand Pharma; he is a member of the Advisory Boards of Eli Lilly, Bristol-Myers Squibb/AstraZeneca, Novo Nordisk and Zealand Pharma, has consulted for AstraZeneca, Gilead Sciences, Ono Pharmaceuticals, Novo Nordisk and Zealand Pharma and received research support from Sanofi. TA owns stocks in Novo Nordisk. LT owns stocks in Novo Nordisk, has received lecture fees and consulted for Novo Nordisk, JJH has consulted for Merck Sharp \& Dome, Novo Nordisk and Roche. SM has participated in advisory boards for Novartis Pharma, Novo Nordisk, Merck Sharp \& Dome, Sanofi, AstraZeneca, Johnson \& Johnson, Roche, Mankind, Boehringer-Ingelheim, Zealand Pharma, Eli Lilly and Intarcia Therapeutics, and has received honoraria for lectures from Novo Nordisk, Merck Sharp \& Dome, Astra-Zeneca, Johnson and Johnson, Roche, Shering-Ploug, Sanofi-Aventis, Novartis Pharma, Eli Lilly and Bristol-Meyer Squibb. HUA owns stocks in Novo Nordisk and participates in an advisory board for Abbott.

Ethics approval The Scientific-Ethical Committee of the Capital Region of Denmark (H-1-2012-031)

Provenance and peer review Not commissioned; externally peer reviewed.

Open Access This is an Open Access article distributed in accordance with the Creative Commons Attribution Non Commercial (CC BY-NC 4.0) license, which permits others to distribute, remix, adapt, build upon this work noncommercially, and license their derivative works on different terms, provided the original work is properly cited and the use is non-commercial. See: http:// creativecommons.org/licenses/by-nc/4.0/

\section{REFERENCES}

1. Banting FG, Best $\mathrm{CH}$. The internal secretion of the pancreas 1922 Indian J Med Res 2007;125:251-66.

2. [No authors listed]. The effect of intensive treatment of diabetes on the development and progression of long-term complications in insulin-dependent diabetes mellitus. The Diabetes Control and Complications Trial Research Group. N Engl J Med 1993;329:977-86.

3. Nathan DM, Cleary PA, Backlund JY, et al. Intensive diabetes treatment and cardiovascular disease in patients with type 1 diabetes. N Engl J Med 2005;353:2643-53

4. American Diabetes Association. Standards of medical care in diabetes-2014. Diabetes Care 2014;37(Suppl 1):S14-80.

5. Cryer PE. Hypoglycemia: still the limiting factor in the glycemic management of diabetes. Endocr Pract 2008;14:750-6.
6. Cryer PE, Davis SN, Shamoon H. Hypoglycemia in diabetes. Diabetes Care 2003;26:1902-12.

7. Must A, Spadano J, Coakley EH, et al. The disease burden associated with overweight and obesity. JAMA 1999;282:1523-9.

8. Wajchenberg BL, Feitosa AC, Rassi N, et al. Glycemia and cardiovascular disease in type 1 diabetes mellitus. Endocr Pract 2008;14:912-23.

9. Chillaron JJ, Flores Le-Roux JA, Benaiges D, et al. Type 1 diabetes, metabolic syndrome and cardiovascular risk. Metabolism 2014;63:181-7.

10. Inzucchi SE, Bergenstal RM, Buse JB, et al. Management of hyperglycemia in type 2 diabetes: a patient-centered approach: position statement of the American Diabetes Association (ADA) and the European Association for the Study of Diabetes (EASD). Diabetes Care 2012;35:1364-79.

11. Russell-Jones D, Khan R. Insulin-associated weight gain in diabetes-causes, effects and coping strategies. Diabetes Obes Metab 2007;9:799-812.

12. Eng C, Kramer CK, Zinman B, et al. Glucagon-like peptide-1 receptor agonist and basal insulin combination treatment for the management of type 2 diabetes: a systematic review and meta-analysis. Lancet 2014;384:2228-34

13. Madsbad S, Krarup T, Deacon CF, et al. Glucagon-like peptide receptor agonists and dipeptidyl peptidase-4 inhibitors in the treatment of diabetes: a review of clinical trials. Curr Opin Clin Nutr Metab Care 2008;11:491-9.

14. Kielgast U, Holst JJ, Madsbad S. Antidiabetic actions of endogenous and exogenous GLP-1 in type 1 diabetic patients with and without residual beta-cell function. Diabetes 2011;60:1599-607.

15. Ghazi T, Rink L, Sherr JL, et al. Acute metabolic effects of exenatide in patients with type 1 diabetes with and without residual insulin to oral and intravenous glucose challenges. Diabetes Care 2014;37:210-16.

16. Gutniak M, Orskov C, Holst JJ, et al. Antidiabetogenic effect of glucagon-like peptide-1 (7-36)amide in normal subjects and patients with diabetes mellitus. N Engl J Med 1992;326:1316-22.

17. Dupre J, Behme MT, Hramiak IM, et al. Glucagon-like peptide reduces postprandial glycemic excursions in IDDM. Diabetes 1995;44:626-30.

18. Hari Kumar KV, Shaikh A, Prusty P. Addition of exenatide or sitagliptin to insulin in new onset type 1 diabetes: a randomized, open label study. Diabetes Res Clin Pract 2013;100:e55-8.

19. Kielgast U, Krarup T, Holst JJ, et al. Four weeks of treatment with liraglutide reduces insulin dose without loss of glycemic control in type 1 diabetic patients with and without residual beta-cell function. Diabetes Care 2011;34:1463-8.

20. Kuhadiya ND, Malik R, Bellini NJ, et al. Liraglutide as additional treatment to insulin in obese patients with type 1 diabetes mellitus. Endocr Pract 2013;19:963-7.

21. Rother KI, Spain LM, Wesley RA, et al. Effects of exenatide alone and in combination with daclizumab on beta-cell function in long-standing type 1 diabetes. Diabetes Care 2009;32:2251-7.

22. Sarkar G, Alattar M, Brown RJ, et al. Exenatide treatment for 6 months improves insulin sensitivity in adults with type 1 diabetes. Diabetes Care 2014;37:666-70.

23. Varanasi A, Bellini N, Rawal D, et al. Liraglutide as additional treatment for type 1 diabetes. Eur J Endocrinol 2011;165:77-84.

24. Kuhadiya N, Dhindsa S, Mehta A, et al. Liraglutide as additional treatment to insulin in patients with type 1 diabetes mellitus: a randomized clinical trial, 23rd Annual Meeting and Clinical Congress of the American Association of Clinical Endocrinologists (AACE), abstract 295, 2014.

25. TK H. NBV: Type 1 Diabetes Mellitus. Secondary NBV: Type 1 Diabetes Mellitus 2014, 2014. http://www.endocrinology.dk/index php/1-diabetes-mellitus/3-type-1-diabetes-mellitus

26. Bergenstal RM, Tamborlane WV, Ahmann A, et al. Effectiveness of sensor-augmented insulin-pump therapy in type 1 diabetes. $N$ Engl $J$ Med 2010;363:311-20.

27. Li L, Shen J, Bala MM, et al. Incretin treatment and risk of pancreatitis in patients with type 2 diabetes mellitus: systematic review and meta-analysis of randomised and non-randomised studies. BMJ 2014;348:g2366.

28. Madsbad S. Prevalence of residual B cell function and its metabolic consequences in Type 1 (insulin-dependent) diabetes. Diabetologia 1983;24:141-7.

29. Unger $\mathrm{RH}$, Aguilar-Parada E, Muller WA, et al. Studies of pancreatic alpha cell function in normal and diabetic subjects. J Clin Invest 1970;49:837-48.

30. Nauck MA, Kemmeries G, Holst JJ, et al. Rapid tachyphylaxis of the glucagon-like peptide 1-induced deceleration of gastric emptying in humans. Diabetes 2011;60:1561-5. 